Radiologe 2013 · 53:539

DOI 10.1007/s00117-013-2509-2

Online publiziert: 18. Mai 2013

c) Springer-Verlag Berlin Heidelberg 2013

\section{J.T. Heverhagen}

Departement Radiologie, Neuroradiologie und Nuklearmedizin, Universitätsinstitut für

Diagnostische, Interventionelle und Pädiatrische Radiologie (DIPR), Inselspital, Universitätsspital Bern, Bern

\section{Leserbrief zum Beitrag}

Reimer O, Vosshenrich R (2013) Kontrastmittel in der Radiologie. Aktuelle Zulassungen, Empfehlungen und Sicherheitshinweise. Radiologe 53:153-164

In ihrem Artikel „Kontrastmittel in der Radiologie" berichten die Autoren über den Einsatz verschiedener Kontrastmittel in allen Bereichen der bildgebenden Diagnostik [1]. Bei ihren Ausführungen stützen sie sich im Wesentlichen auf die aktuelle Leitlinie der European Society of Urogential Radiology (ESUR) 8.0 [2]. Leider sind den Autoren bei der Abfassung des Manuskripts einige Ungenauigkeiten, die aber für die tägliche Praxis von großer Bedeutung sein können, unterlaufen, die ich mit diesem Leserbrief korrigieren möchte.

Zunächst beschreiben die Autoren in Tab. 3 die z. Z. auf dem Markt befindlichen gadoliniumhaltigen Präparate korrekt. Allerdings ist hier zu erwähnen, dass für das erste Präparat, Magnevist, die $\mathrm{Zu}$ lassung nicht ab Geburt, sondern erst bei Säuglingen ab einem Alter von 4 Wochen gilt. Bei jüngeren Säuglingen ist es sogar kontraindiziert [3]. In der gleichen Tabelle fehlt die Indikation für die MR-Angiographie für Dotarem [4]. Auf Seite 156 beschreiben die Autoren, dass für eine wiederholte Anwendung gadoliniumhaltiger Kontrastmittel (Gd-KM) bei Kindern ein Abstand von mindestens 7 Tagen zwischen den Injektionen liegen sollte. Dies gilt nach den ESUR-Leitlinien allerdings nur für Kinder, welche jünger als ein Jahr sind, und für Patienten mit eingeschränkter Nierenfunktion [2].

In den Tab. 6, 7 und 8 beschreiben die Autoren die Zuordnung der verschiedenen Gd-KM zu den NSF-Risikogruppen nach ESUR korrekt. Es gibt die Gruppe mit hohem NSF-Risiko mit den Kontrastmitteln Omniscan, Magnevist und Optimark, die Gruppe mit mittlerem Risiko mit den Kontrastmitteln Multihance, Vasovist (Ablavar) und Primovist (Eovist) und die Gruppe mit niedrigem Risiko mit Gadovist (Gadavist), Dotarem (Magnescope) und Prohance. Dabei unterscheiden sich die Gruppen mit mittlerem und niedrigem NSF-Risiko weder in der Inzidenz der NSF-Fälle noch in den Empfehlungen der ESUR. In Tab. 7 wird nun beschrieben, dass für alle Kontrastmittel der mittleren Risikogruppe bisher keine eindeutigen Fälle beschrieben wurden, und dass die Erfahrungen mit diesen Präparaten limitiert sind. Nach den ESUR-Leitlinien sind die Erfahrungen allerdings nur mit Vasovist (Ablavar) und Primovist (Eovist) limitiert. Für Multihance liegen gemäß der ESUR-Leitlinien hingegen keine eindeutigen NSF-Fälle bei ausreichenden Erfahrungen vor. In Tab. 8 steht, dass sowohl für Gadovist (Gadavist) als auch für Dotarem einige unklare Fälle berichtet wurden, deren Histopathologie unsicher ist. Dies gilt allerdings nur für Gadovist (Gadavist). Für Dotarem sind gemäß ESUR bisher keine eindeutigen Fälle beschrieben.

Bezüglich Schwangerschaft und Stillzeit geben die ESUR-Leitlinien ebenfalls eindeutige Empfehlungen. Bei einer zwingenden Indikation für eine kontrastverstärkte MR-Untersuchung sollte Schwangeren die kleinstmögliche Dosis eines der stabilsten Kontrastmittel (mittlere oder niedrige Risikogruppe) gegeben werden. Die Autoren haben hier im Gegensatz zur ESUR-Leitlinie die Verwendung ausschließlich makrozyklischer Substanzen empfohlen. Ist bei stillenden Müt- tern eine kontrastverstärkte MR-Untersuchung notwendig, ist eine Stillunterbrechung für $24 \mathrm{~h}$ nach Kontrastmittelgabe nur bei Verwendung eines Kontrastmittels der Gruppe mit hohem NSF-Risiko empfohlen. Für alle anderen Kontrastmittel sollte die Patientin vom Radiologen ausführlich informiert werden, um dann gemeinsam zu entscheiden, ob eine Stillunterbrechung von $24 \mathrm{~h}$ notwendig ist.

Ich hoffe, mit diesen Klarstellungen den sehr informativen und ausführlichen Artikel „Kontrastmittel in der Radiologie" noch etwas klarer praxisnäher gestaltet zu haben.

\section{Korrespondenzadresse}

Prof. Dr. Dr. J.T. Heverhagen

Departement Radiologie, Neuroradiologie und Nuklearmedizin, Universitätsinstitut für Diagnostische, Interventionelle und Pädiatrische Radiologie (DIPR), Inselspital, Universitätsspital Bern,

Freiburgstr. 10, CH-3010 Bern

Johannes.Heverhagen@insel.ch

Interessenkonflikt. Der korrespondierende Autor weist auf folgende Beziehungen hin: Drittmittel von Bracco SpA und Bayer Schering Pharma AG und Guerbet AG; Referententätigkeit: Bracco SpA.

\section{Literatur}

1. Reimer P, Vosshenrich R (2013) Contrast agents in radiology: current agents approved, recommendations, and safety aspects. Radiologe 53:153-164

2. Thomsen HS, Webb JA (2012) ESUR guidelines on contrast media, Version 8.0. European Society of Urogenital Radiology, Wien. http://wwwesurorg/ guidelines/. Zugegriffen: 02. Apr. 2013

3. Bayer Schering AG (2011) Fachinformation Magnevist $0,5 \mathrm{mmol} / \mathrm{ml}$, Injektionslösung

4. Guerbet AG (2010) Fachinformation Dotarem 